\title{
THE PREDUAL THEOREM TO THE JACOBSON-BOURBAKI THEOREM
}

\author{
BY \\ MOSS SWEEDLER( ${ }^{1}$ )
}

ABSTRACT. Suppose $R \stackrel{\varphi}{\longrightarrow} S$ is a map of rings. $S$ need not be an $R$ algebra since $R$ may not be commutative. Even if $R$ is commutative it may not have central image in $S$. Nevertheless the ring structure on $S$ can be expressed in terms of two maps

$$
s \otimes_{R} s \stackrel{\left(s_{1} \otimes s_{2} \rightarrow s_{1} s_{2}\right)}{\longrightarrow} s, \quad R \stackrel{\varphi}{\rightarrow} s,
$$

which satisfy certain commutative diagrams. Reversing all the arrows leads to the notion of an $R$-coring.

Suppose $R$ is an overing of $B$. Let $C_{B}=R \otimes_{B} R$. There are maps

$$
\begin{aligned}
& C_{B}=R \otimes_{B} R \stackrel{\left(r_{1} \otimes r_{2} \rightarrow r_{1} \otimes 1 \otimes r_{2}\right)}{\longrightarrow} R \otimes_{B} R \otimes_{B} R=\left(C_{B}\right) \otimes_{R}\left(C_{B}\right), \\
& C_{B}=R \otimes_{B} R \stackrel{\left(r_{1} \otimes r_{2} \rightarrow r_{1} r_{2}\right)}{\longrightarrow} R .
\end{aligned}
$$

These maps give $C_{B}$ an $R$-coring structure. The dual ${ }^{*} C_{B}$ is naturally isomorphic to the ring End ${ }_{B}-R$ of $B$-linear endomorphisms of $R$ considered as a left $B$-module. In case $B$ happens to be the subring of $R$ generated by 1 , we write $C_{\mathrm{Z}}$. Then ${ }^{*} C_{\mathrm{Z}}$ is End $\mathrm{Z}^{R}$, the endomorphism ring of $R$ considered as an additive group. This gives a clue how certain $R$-corings correspond to subrings of $R$ and subrings of End $R$, both major ingredients of the Jacobson-Bourbaki theorem.

$1 \otimes 1$ is a "grouplike" element in the $R$-coring $C_{Z}$ (and should be thought of as a generic automorphism of $R$ ). Suppose $R$ is a division ring and $B$ a subring which is a division ring. The natural map $C_{Z} \rightarrow C_{B}$ is a surjective coring map. Conversely if $C_{Z} \stackrel{\pi}{\rightarrow} D$ is a (surjective) coring map then $\pi(1 \otimes 1)$ is a grouplike in $D$ and $\{r \in R \mid r \pi(1 \otimes 1)=\pi(1 \otimes 1) r\}$ is a subring of $R$ which is a division ring. This gives a bijective correspondence between the quotient corings of $C \otimes_{\mathrm{Z}} C$ and the subrings of $R$ which are division rings.

We show how the Jacobson-Bourbaki correspondence is dual to the above correspondence.

Received by the editors December 17, 1974.

AMS (MOS) subject classifications (1970). Primary 16A74; Secondary 16A40, 16A56. Key words and phrases. Coring, coalgebra, division ring.

(1) Supported in part by NSF Grant GP 40773X. 
Another viewpoint. $B \otimes_{\mathrm{Z}} B$ is a $B$-bimodule. For a subring $A \subset B, K=$ $\operatorname{ker}\left(B \otimes_{\mathrm{Z}} B \rightarrow B \otimes_{A} B\right)$ is a sub-B-bimodule of $B \otimes_{\mathrm{Z}} B$.

$K$ is generated by $\{a \otimes 1-1 \otimes a\}_{a \in A}$ as a $B$-bimodule. There are sub-Bbimodules not of this form.

$K$, as above, lies in

$$
\operatorname{kernel}\left(B \otimes_{\mathrm{Z}} B \stackrel{\text { multiplication }}{\longrightarrow} B\right),
$$

and if $e_{1}: B \otimes_{\mathrm{Z}} B \rightarrow B \otimes_{\mathrm{Z}} B \otimes_{\mathrm{Z}} B, \Sigma b_{i} \otimes \beta_{i} \rightarrow \Sigma b_{i} \otimes 1 \otimes \beta_{i}$, then

$$
e_{1}(K) \subset B \otimes_{\mathrm{Z}} K+K \otimes_{\mathrm{Z}} B \text {. }
$$

(*) and (**) amount to the same thing as saying that $K$ is a coideal. Thus the language of corings merely gives us a convenient way of describing the sub$B$-bimodules of $B \otimes_{\mathrm{Z}} B$ generated by $\{a \otimes 1-1 \otimes a\}_{a \in A}$.

Infinite duality. The theory as we have developed it with $B \otimes_{Z} B$ as a coring can be dualized in general. The dual to $B \otimes_{\mathrm{Z}} B$ is End $B$. However when there are not finiteness assumptions-in the dual theorems-closedness assumptions and considerations of linear compactness appear. This duality has been worked out by Dieudonné [2].

1. Corings over general rings. If $A$ is a commutative ring, an $A$-coalgebra is an $A$-module $C$ together with maps $\Delta: C \rightarrow C \otimes_{A} C, \epsilon: C \rightarrow A$ having certain properties [7, p. 4]. Since $A$ is commutative there is no real difference between left and right $A$-modules and " $C \otimes_{A} C$ " is well defined. If $A$ were not commutative , then $C \otimes_{A} C$ is only well defined if $C$ has both a left and right $A$-module structure and the tensor product is with respect to the right $A$-module structure of the left $C$ and the left $A$-module structure of the right $C$.

Following Cartan-Eilenberg, we use ${ }_{A} M$ to indicate that $M$ is a left $A$ module and $M_{A}$ indicates that $M$ is a right $A$-module. If $B$ is another ring, we use ${ }_{A} M_{B}$ to indicate that ${ }_{A} M, M_{B}$ and the following condition is satisfied:

$$
(a m) b=a(m b), \quad a \in A, m \in M, b \in B .
$$

$M$ is an $A$-bimodule means " ${ }_{A} M_{A}$ " and not simply " ${ }_{A} M$ and $M_{A}$ ". If $M$ is an $A$-bimodule, and we write $M \otimes_{A} M$, this is always the tensor product $\left(M_{A}\right) \otimes_{A}$ $\left({ }_{A} M\right)$. The natural $A$-bimodule structure on $M \otimes_{A} M$ is determined by

$$
a \cdot\left(\sum m_{i} \otimes m_{i}^{\prime}\right) \cdot b \equiv \sum\left(a m_{i}\right) \otimes\left(m_{i}^{\prime} b\right), \quad a, b \in A, \sum m_{i} \otimes m_{i}^{\prime} \in M \otimes_{A} M .
$$

The natural $A$-bimodule structure on $A$ itself is the one induced by left and right multiplication.

1.1. Definition. For a not necessarily commutative ring $A$, an $A$-coring 
is an $A$-bimodule $M$ together with $A$-bimodule maps $\Delta: M \rightarrow M \otimes_{A} M, \epsilon: M \rightarrow$ $A$ satisfying the following three commutative diagrams:
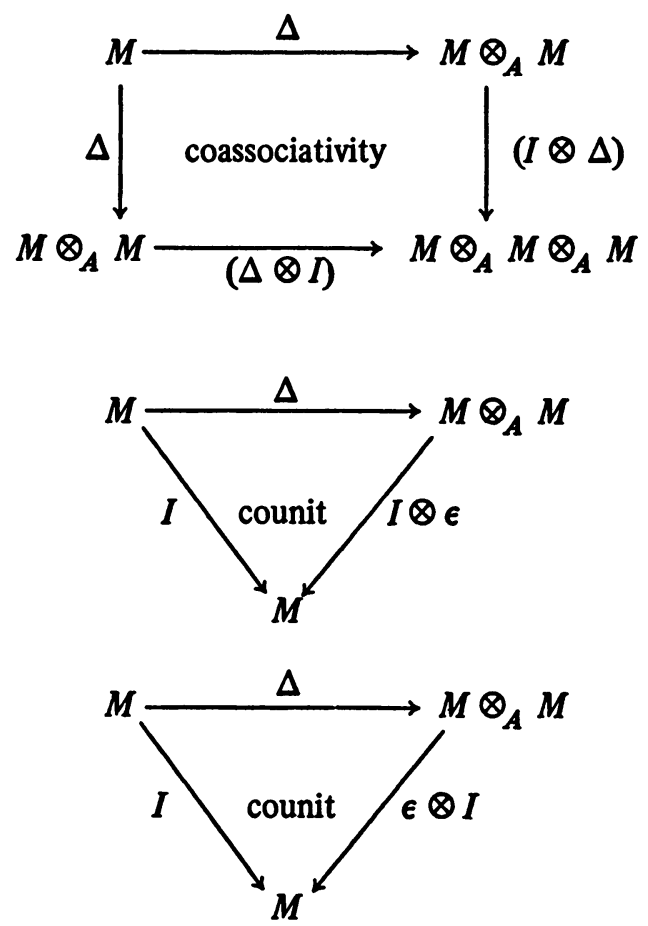

1.2. Example. This is the main example. Suppose $A$ and $B$ are not necessarily commutative rings and $\varphi: A \rightarrow B$ a ring map. The natural $B$-bimodule structure on $B$ gives rise to an $A$-bimodule structure on $B$ via $\varphi$. Form $B \otimes_{A} B$ and call this $M$. The natural $B$-bimodule structure on $M$ is determined by

$$
b \cdot(c \otimes d) \cdot e \equiv(b c) \otimes(d e), \quad b, c, d, e \in B .
$$

Then $M \otimes_{B} M=\left(B \otimes_{A} B\right) \otimes_{B}\left(B \otimes_{A} B\right)$ which is naturally isomorphic to $B \otimes_{A} B \otimes_{A} B$. The (Amitsur complex) map

$$
e_{1}: B \otimes_{A} B \rightarrow B \otimes_{A} B \otimes_{A} B, \quad b \otimes \beta \rightarrow b \otimes 1 \otimes \beta,
$$

determines $\Delta$ via the commutative diagram

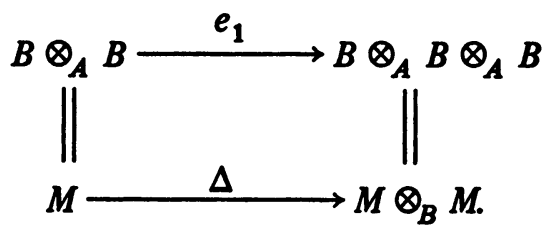

The multiplication map

$$
B \otimes_{A} B \rightarrow B, \quad b \otimes \beta \rightarrow b \beta
$$


determines $\epsilon$ via the commutative diagram

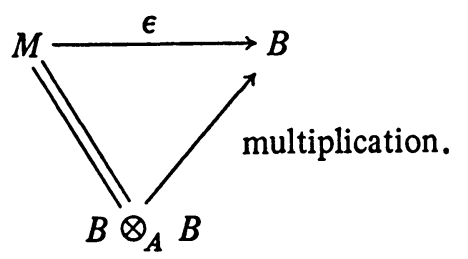

It is left to the reader to verify that $(M, \Delta, \epsilon)$ is a $B$-coring in the sense of (1.1).

1.3. Definition. If $(M, \Delta, \epsilon)$ and $\left(M^{\prime}, \Delta^{\prime}, \epsilon^{\prime}\right)$ are $A$-corings, then a coring map $g: M \rightarrow M^{\prime}$ is an $M$-bimodule map where $\epsilon^{\prime} g=\epsilon$ and $\Delta^{\prime} g=(g \otimes g) \Delta$. A coideal (of $M$ ) is a sub- $A$-bimodule $J \subset M$ where $J \subset$ ker $\epsilon$ and

$$
\Delta\left(J \subset \operatorname { k e r } \left(M \otimes_{A} M \stackrel{\pi \otimes \pi}{\longrightarrow}\left(M / J \otimes_{A}(M / J)\right) .\right.\right.
$$

Here $\pi$ is the canonical $A$-bimodule map $M \rightarrow M / J$. Of course ker $\pi \otimes \pi$ is

$$
\operatorname{Im}\left(J \otimes_{A} M \stackrel{\iota \otimes I}{\longrightarrow} M \otimes_{A} M\right)+\operatorname{Im}\left(M \otimes_{A} J \stackrel{I \otimes \iota}{\longrightarrow} M \otimes_{A} M\right),
$$

where $\iota$ is the natural inclusion $J \rightarrow M$.

As in classical coalgebra theory, if $J$ is a coideal of $M$, then $M / J$ has a unique $A$-coring structure whereby $\pi: M \rightarrow M / J$ is a coring map. Moreover, the kernel of a coring map is a coideal and the expected isomorphism theorem holds. (A source for elementary coalgebra theory is [7, pp. 3-48].)

1.4. DEFinition. If $R$ and $S$ are rings and ${ }_{R} M_{S},{ }_{R} N_{S}$, then $\operatorname{Hom}_{R-S}(M, N)$ is used to denote the maps from $M$ to $N$ which are simultaneously left $R$-module maps and right $S$-module maps. Let $\operatorname{Hom}_{R-}(M, N)$ denote the left $R$-module maps from $M$ to $N$. Let $\operatorname{Hom}_{-S}(M, N)$ denote the right $S$-module maps from $M$ to $N$. If $L$ is an $A$-bimodule let ${ }^{*} L^{*}$ denote $\operatorname{Hom}_{A-A}(L, A)$.

1.5. Proposition. Suppose $(M, \Delta, \epsilon)$ is an $A$-coring. Then ${ }^{*} M^{*}$ has a ring structure where, for $f, g \in{ }^{*} M^{*}$, the product $f g$ is the composite

$$
M \stackrel{\Delta}{\longrightarrow} M \otimes_{A} M \stackrel{f \otimes g}{\longrightarrow} A \otimes_{A} A=A
$$

and the unit of $* M^{*}$ is $\epsilon$.

Proof. Similar to the construction of the dual algebra in classical coalgebra theory [7, pp. 6-10], and left to the reader. Q.E.D.

It is easily verified that if $M$ and $M^{\prime}$ are $A$-corings and $F: M \rightarrow M^{\prime}$ a coring map, then the induced map ${ }^{*} F^{*}:{ }^{*} M^{*} \rightarrow{ }^{*} M^{*}$ is a ring map.

1.6. ExAmple. Let $M$ be the $B$-coring of Example 1.2 so $M=B \otimes_{A} B$. Any $f \in{ }^{*} M^{*}$ is determined by $f(1 \otimes 1)$. If $f(1 \otimes 1)=x \in B$, then $a \in A$, 
$a x=a f(1 \otimes 1)=f(a \otimes 1)=f(1 \otimes a)=f(1 \otimes 1) a=x a$.

Conversely if $x$ lies in the centralizer of $\varphi(A)$, then $B \otimes_{A} B \rightarrow B, b \otimes \beta$

$\rightarrow b x \beta$ gives a $B$-bimodule map $g$ with $g(1 \otimes 1)=x$. Hence $* M^{*}$ is naturally isomorphic to the centralizer of $\varphi(A)$ in $B$.

1.7. Definition. If $(M, \Delta, \epsilon)$ is an $A$-coring, then $g \in M$ is called grouplike if $\epsilon(g)=1$ and $\Delta(g)=g \otimes g$.

A grouplike cannot be zero since $\epsilon(g)=1$.

1.8. Definition. The trivial $A$-coring structure on $A$ is the $A$-coring $(A, \Delta, \epsilon)$ where $\Delta$ and $\epsilon$ are determined by the commutative diagrams:
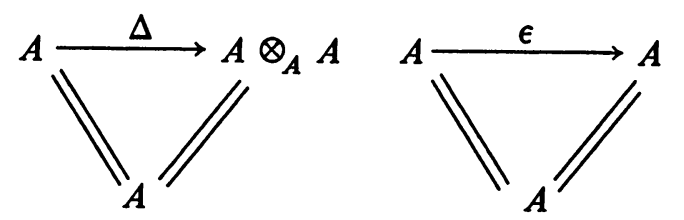

If $A$ has the trivial $A$-coring structure, then 1 is a grouplike.

1.9. Proposition. Suppose $A$ and $B$ are rings and $\varphi: A \rightarrow B$ a ring map. Let $M=B \otimes_{A} B$ have the $B$-coring structure of 1.2 .

(a) Let $g_{B / A}$ denote $1 \otimes 1 \in B \otimes_{A} B ; g_{B / A}$ is a grouplike element of $M$.

(b) Let $C$ be a $B$-coring and let $\mathbf{G}_{\varphi}(C)$ denote $\{g \in C \mid g$ is grouplike and $\varphi(a) g=g \varphi(a), a \in A\}$.

For each $g \in \mathrm{G}_{\varphi}(C)$ there is a unique coring map $\zeta: M \rightarrow C$ with $\zeta\left(g_{B / A}\right)$ $=g$.

For each coring map $\xi: M \rightarrow C$, the element $\xi\left(g_{B / A}\right) \in \mathrm{G}_{\varphi}(C)$.

Proof. Left to the reader. Q.E.D.

2. Correspondence theorem.

2.1. Fundamental Theorem. Suppose $A \subset B$ are division rings. Let $M$ be the B-coring formed by $A \hookrightarrow B$ as in 1.2 and let $C$ denote the set of coideals of $M$. Let $D$ denote the set of (intermediate) division rings $D$ with $A \subset D \subset B$.

(a) For $D \in D$ the kernel of the natural surjective map $M=B \otimes_{A} B \rightarrow$ $B \otimes_{D} B$ is a coideal. Denote it by $J_{D}$.

(b) For a coideal $J \in C$, let $\pi: M \rightarrow M / J$ be the natural surjective map. Then $\left\{b \in B \mid b \pi\left(g_{B / A}\right)=\pi\left(g_{B / A}\right) b\right\}$ is a division ring intermediate between $A$ and $B$. Denote it by $D_{J}$.

(c) The maps

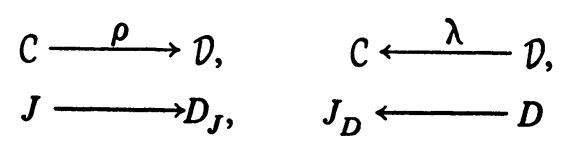

are inverse to each other, thus establishing a bijective correspondence. 
Proof. (a) Let $N$ be the $B$-coring formed by $D \hookrightarrow B$ as in 1.2. Then the natural map $M=B \otimes_{A} B \rightarrow B \otimes_{D} B=N$ is a coring map and so has a kernel which is a coideal.

(b) Let $L$ be any $B$-bimodule and $l \in L$. If $b l=l b$, then

$$
b^{-1} l=b^{-1} l 1=b^{-1} l b b^{-1}=b^{-1} b l b^{-1}=1 l b^{-1}=l b^{-1} .
$$

Hence $\{b \in B \mid b l=l b\}$ is a subring of $B$ which is a division ring.

For $g_{B / A}=1 \otimes 1 \in B \otimes_{A} B, a g_{B / A}=g_{B / A} a$ for all $a \in A$. Since $\pi: M \rightarrow$ $M / J$ is a $B$-bimodule map, it follows that $a \pi\left(g_{B / A}\right)=\pi\left(g_{B / A}\right) a$ for all $a \in A$. Hence $\left\{b \in B \mid b \pi\left(g_{B / A}\right)=\pi\left(g_{B / A}\right) b\right\}$ is a subring of $B$ which is a division ring and contains $A$.

(c) $(\rho \lambda=I)$ Suppose $D \in D$. Then $J_{D}$ is the kernel of the natural map $\pi: B \otimes_{A} B \rightarrow B \otimes_{D} B$ and $\pi\left(g_{B / A}\right)=1 \otimes 1 \in B \otimes_{D} B$. Hence

$$
\begin{aligned}
D_{J_{D}} & =\left\{b \in B \mid b(1 \otimes 1)=(1 \otimes 1) b \in B \otimes_{D} B\right\} \\
& =\left\{b \in B \mid(b \otimes 1)=(1 \otimes b) \in B \otimes_{D} B\right\} .
\end{aligned}
$$

For $e \in B$ with $e \notin D$, the set $\{1, e\}$ is $D$-linearly independent when $B$ is considered as a left $D$-module. Hence for $x, y \in B$, if $0=x \otimes 1+y \otimes e \in B \otimes_{D} B$, then both $x$ and $y$ must equal zero. In particular, $0 \neq(-e) \otimes 1+1 \otimes e$, which proves that $D_{J_{D}} \subset D$. The opposite inclusion is obvious and hence $D_{J_{D}}=D$.

$(\lambda \rho=I)$ Let $J$ be a coideal of $M$, and $\pi: M \rightarrow M / J$ the natural coring map. By 1.9 there is a unique coring map $\zeta: B \otimes_{D_{J}} B \rightarrow M / J$ with $\zeta\left(g_{B / D_{J}}\right)=\pi\left(g_{B / A}\right)$. We have the commutative diagram

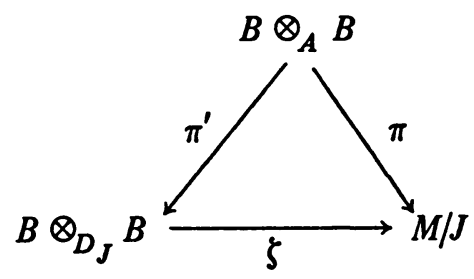

where $\pi^{\prime}$ is the natural surjection. (Note, the diagram must commute since $\zeta \pi^{\prime}\left(g_{B / A}\right)=\zeta\left(g_{B / D_{J}}\right)=\pi\left(g_{B / A}\right)$ and $B \otimes_{A} B$ is generated by $g_{B / A}$ as a $B$-bimodule and all the maps are $B$-bimodule maps.)

The coring $B \otimes_{A} B$ is generated by $g_{B / A}$ as a $B$-bimodule; hence, the quotient $M / J$ is generated by the grouplike $\pi\left(g_{B / A}\right)$ as a $B$-bimodule. By definition,

$$
D_{J}=\left\{b \in B \mid b \pi\left(g_{B / A}\right)=\pi\left(g_{B / A}\right) b\right\} .
$$

Hence by the following lemma (2.2), the map $\zeta$ is a coring isomorphism. From the commutative diagram we deduce $\operatorname{ker} \pi^{\prime}=\operatorname{ker} \pi$. Hence $J_{D_{J}}=\operatorname{ker} \pi^{\prime}=$ ker $\pi=J$. Q.E.D. 
2.2. Fundamental Lemma. Let $B$ be a division ring and $C a$-coring. Suppose $g$ is a grouplike element of $C$ and $D=\{b \in B \mid b g=g b\}$. Then $D$ is $a$ subring of $B$ which is a division ring, and the unique coring map from 1.11 , with $\zeta(1 \otimes 1)=C, \zeta: B \otimes_{D} B \rightarrow C$, is injective. $\zeta$ is an isomorphism if $C$ is generated by $g$ as a B-bimodule.

Proof. That $D$ is a division ring follows from the first few lines in the proof of 2.1(b).

Explicitly the map $\zeta: B \otimes_{D} B \rightarrow C$ is given by $\Sigma b_{i} \otimes \beta_{i} \stackrel{\zeta}{\rightarrow} \Sigma b_{i} g \beta_{i},\left\{b_{i}\right\} \cup$ $\left\{\beta_{i}\right\} \subset B$. Suppose ker $\zeta \neq 0$. Choose a nonzero element $x=\Sigma^{t} b_{i} \otimes \beta_{i} \in \operatorname{ker} \zeta$ with $t$ minimal.

$t \neq 1$ : if $x=b_{1} \otimes \beta_{1} \in \operatorname{ker} \zeta$, then $b_{1} g \beta_{1}=0$. Since $g \neq 0$ by the remark following 1.9 , and since $B$ is a division ring, it follows that $b_{1}$ or $\beta_{1}$ must equal zero. This contradicts the fact that $x \neq 0$.

$t \ngtr 1$ : Since $t$ is minimal it follows that $\beta_{t} \neq 0$. The element $0 \neq x^{\prime}=$ $\Sigma^{t} b_{i} \otimes \beta_{i} \beta_{t}^{-1}$ still lies in ker $\zeta$ and has the same rank $t$. Let $\beta_{i}^{\prime}=\beta_{i} \beta_{t}^{-1}$, so that $\beta_{t}^{\prime}=1$. Since $x^{\prime}=\Sigma^{t} b_{i} \otimes \beta_{i}^{\prime}$, and this is a minimal length expression for $x^{\prime}$, it follows that

(a) $\left\{b_{i}\right\}$ is a $D$-linearly independent set in $B_{D}$,

(b) $\left\{\beta_{i}^{\prime}\right\}$ is a $D$-linearly independent set in ${ }_{D} B$.

Hence since $\beta_{t}^{\prime}=1 \in D$, it follows from (b) that $\beta_{1}^{\prime} \notin D$.

Thus $g \beta_{1}^{\prime} \neq \beta_{1}^{\prime} g$, and there is $h: C \rightarrow B$ a left $B$-module map (i.e. $h(b z)=$ $b h(z), b \in B, z \in C)$ with $h\left(g \beta_{1}^{\prime}-\beta_{1}^{\prime} g\right) \neq 0$. Such $h$ exists because as a left $B$ module $C$ is free; $B$ is a division ring after all.

Let $x^{\prime \prime}=\Sigma^{t} b_{i} \otimes h\left(g \beta_{i}^{\prime}-\beta_{i}^{\prime} g\right)$. Then,

(c) since $\beta_{t}^{\prime}=1, g \beta_{t}^{\prime}=\beta_{t}^{\prime} g$ and $x^{\prime \prime}=\Sigma^{t-1} b_{i} \otimes h\left(g \beta_{i}^{\prime}-\beta_{i}^{\prime} g\right)$, i.e. $x^{\prime \prime}$ has an expression of length less than $t$.

(d) $0 \neq x^{\prime \prime}$, since $0 \neq h\left(g \beta_{1}^{\prime}-\beta_{1}^{\prime} g\right)$ and $\left\{b_{i}\right\}$ is a $B_{D}, D$-linearly independent set.

(e) $\zeta\left(x^{\prime \prime}\right)=\sum^{t} b_{i} g h\left(g \beta_{i}^{\prime}-\beta_{i}^{\prime} g\right)=\sum^{t} b_{i} g h\left(g \beta_{i}^{\prime}\right)-\sum^{t} b_{i} g h\left(\beta_{i}^{\prime} g\right)$.

The term

$$
\sum^{t} b_{i} g h\left(\beta_{i}^{\prime} g\right)=\sum^{t} b_{i} g \beta_{i}^{\prime} h(g)=\underbrace{\zeta\left(x^{\prime}\right)}_{0} h(g)=0 .
$$

The term $\Sigma^{t} b_{i} g h\left(g \beta_{i}^{\prime}\right)$ is the image of $x^{\prime}$ under the composite

$$
B \otimes_{A} B \stackrel{\zeta}{\longrightarrow} C \stackrel{\Delta}{\longrightarrow} C \otimes_{B} C \stackrel{I \otimes h}{\longrightarrow} C \otimes_{B} B=C,
$$

but

Hence $x^{\prime \prime} \in \operatorname{ker} \zeta$.

$$
(I \otimes h) \Delta \underbrace{\left(x^{\prime}\right)}_{0}=0 .
$$


But (c), (d) and (e) contradict the minimality of $t$. Hence ker $\zeta=0$.

Since $\operatorname{Im} \zeta$ is the sub-B-bimodule of $C$ generated by $g$, it follows that $\zeta$ is an isomorphism when $C=B g B$. Q.E.D.

2.3. CoROllary. Suppose $B$ is a division ring and $P$ is the prime field contained in $B$. So that $P \cong Z / p Z$ if $B$ has characteristic $p$ and $P \cong Q$ if $B$ has characteristic zero. Then the correspondence in 2.1 gives a bijective correspondence between the set of all coideals in $B \otimes_{P} B$ on the one hand and the set of all subdivision rings of $B$ on the other hand.

3. Semiduality. This section consists of technical, boring, but important results on duality. The reader is advised to skip to $\$ 4$ and refer to this section as needed.

3.1. Definition. If $M$ is a $B$-bimodule let $M^{*}$ denote $\operatorname{Hom}_{-B}(M, B)$, the set of right $B$-module maps from $M$ to $B$, and let $* M$ denote $\operatorname{Hom}_{B-}(M, B)$, the set of left $B$-module maps from $M$ to $B$.

The picture is:

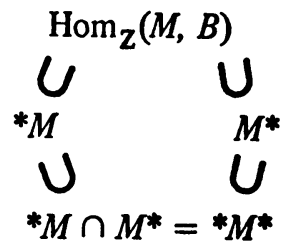

3.2. Proposition. Suppose $(C, \Delta, \epsilon)$ is a $B$-coring.

(a) ${ }^{*} C$ has a ring structure where, for $f, g \in{ }^{*} C$, the product $g f$ is the composite

$$
C \stackrel{\Delta}{\longrightarrow} C \otimes_{B} C \stackrel{I \otimes f}{\longrightarrow} C \otimes_{B} B=C \stackrel{g}{\longrightarrow} B,
$$

and the unit of ${ }^{*} C$ is $\epsilon$.

(b) $C^{*}$ has a ring structure where, for $f, g \in C^{*}$, the product $g f$ is the composite

$$
C \stackrel{\Delta}{\longrightarrow} C \otimes_{B} C \stackrel{g \otimes I}{\longrightarrow} B \otimes_{B} C=C \stackrel{f}{\longrightarrow} B,
$$

and the unit of $C^{*}$ is $\epsilon$.

(c) The map $\lambda: B \rightarrow{ }^{*} C$, determined by $\lambda(b)(c)=\epsilon(c b), b \in B, c \in C$, is a ring antimap, i.e. reverses multiplication

(d) The map $\rho: B \rightarrow C^{*}$, determined by $\rho(b)(c)=\epsilon(b c), b \in B, c \in C$, is a ring antimap.

If $C^{\prime}$ is another $B$-coring and $\Xi: C \rightarrow C^{\prime}$ a B-coring map then:

(e) The natural map ${ }^{*}\left(C^{\prime}\right) \stackrel{{ }^{*} \Xi}{\longrightarrow}{ }^{*} C$ is a ring map making the diagram commute: 


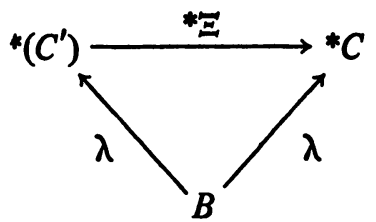

(f) The natural map $\left(C^{\prime}\right)^{*} \stackrel{\Xi^{*}}{\longrightarrow} C^{*}$ is a ring map making the diagram commute:

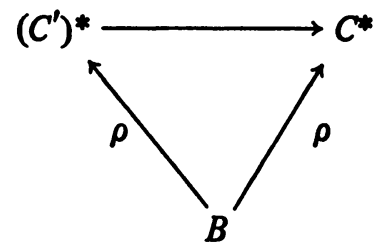

Proof. (a) For $f, g, h \in{ }^{*} C$ the product $h(g f)$ or $(h g) f$ both are the composite

$$
\begin{aligned}
C \stackrel{\Delta_{2}}{\longrightarrow} C \otimes_{B} C \otimes_{B} C \stackrel{I \otimes I \otimes f}{\longrightarrow} C \otimes_{B} C \otimes_{B} B \\
=C \otimes_{B} C \stackrel{I \otimes g}{\longrightarrow} C \otimes_{B} B=C \stackrel{h}{\longrightarrow} B,
\end{aligned}
$$

where $\Delta_{2}$ is either of the composites

$$
\begin{aligned}
& C \stackrel{\Delta}{\longrightarrow} C \otimes_{B} C \stackrel{I \otimes \Delta}{\longrightarrow} C \otimes_{B} C \otimes_{B} C, \\
& C \stackrel{\Delta}{\longrightarrow} C \otimes_{B} C \stackrel{\Delta \otimes I}{\longrightarrow} C \otimes_{B} C \otimes_{B} C
\end{aligned}
$$

which are the same by coassociativity.

The rest of the ring axioms are easily verified.

(b) Similar to (a).

The other verifications are left to the reader. Q.E.D.

3.3. ExAMPLE. Suppose $B$ is a ring with subring $A$. We have the inclusion $A \hookrightarrow B$ and form $M=B \otimes_{A} B$ as in 1.2. The natural correspondence

$(* *)$

$$
{ }^{*} M=\operatorname{Hom}_{B-}\left(B \otimes_{A} B, B\right)=\operatorname{Hom}_{A-}(B, B)=\operatorname{End}_{A-} B
$$

is given as follows: $f \in{ }^{*} M$ corresponds to $B \rightarrow B, b \rightarrow f\left(g_{B / A} b\right)$ where $g_{B / A}=$ $1 \otimes 1 \in B \otimes_{A} B$ the grouplike element of 1.9 .

It is left to the reader to show that the above correspondence $* M=$ End $_{A-B} B$ is a ring isomorphism.

Similarly the natural correspondence

$$
M^{*}=\operatorname{Hom}_{-B}\left(B \otimes_{A} B, B\right)=\operatorname{Hom}_{-A}(B, B)=\text { End }_{-A} B
$$

is given as follows: $f \in M^{*}$ corresponds to $B \rightarrow B, b \rightarrow f\left(b g_{B / A}\right)$. It is left to 
the reader to verify that the correspondence $M^{*}=$ End $_{-A} B$ is a ring anti-isomorphism.

3.4. LineAR LEMMA. Suppose $X$ and $Y$ are rings and there are modules ${ }_{Y} R,{ }_{X} S_{Y}, T_{X}, U_{Y}, X$.

(a) The right $X$-module structure on $\operatorname{Hom}_{-Y}(S, U)$ arises from ${ }_{X} S$ and the left $X$-module structure on $\mathrm{Hom}_{-X}(T, X)$ arises from ${ }_{X} X$.

There is a natural map

$$
\eta: \mathrm{Hom}_{-Y}(S, U) \otimes_{X} \mathrm{Hom}_{-X}(T, X) \rightarrow \mathrm{Hom}_{-Y}\left(T \otimes_{X} S, U\right)
$$

for $f \in \mathrm{Hom}_{-Y}(S, U), g \in \mathrm{Hom}_{-X}(T, X), t \in T, s \in S$,

$$
\eta(f \otimes g)(t \otimes s)=f(g(t) s)
$$

$\eta$ is bijective if $T$ is a finitely generated projective right $X$-module.

(b) The left $Y$-module structure on $\operatorname{Hom}_{X-}(S, V)$ arises from $S_{Y}$ and the right $X$-module structure on $\operatorname{Hom}_{Y-}(R, Y)$ arises from $Y_{Y}$.

There is a natural map

$$
\mu: \operatorname{Hom}_{Y-}(R, Y) \otimes_{Y} \operatorname{Hom}_{X-}(S, V) \rightarrow \operatorname{Hom}_{X-}\left(S \otimes_{Y} R, V\right) ;
$$

for $f \in \operatorname{Hom}_{Y_{-}}(R, Y), g \in \operatorname{Hom}_{X-}(S, V), s \in S, r \in R$,

$$
\mu(f \otimes g)(s \otimes r)=g(s f(r))
$$

$\mu$ is bijective if $R$ is a finitely generated projective left $Y$-module.

Suppose $W$ and $Z$ are also rings and we have the additional hypothesis: ${ }_{Y} R_{W}, T_{X},{ }_{W} U_{Y}, V_{Z}$.

(c) If the domain and range of $\eta$ have the left $W$-module structure arising from ${ }_{W} U$ and the right $Z$-module structure arising from ${ }_{z} T$, then $\eta$ is a left $W$ module map and right Z-module map.

(d) If the dọmain and range of $\mu$ have the left $W$-module structure arising from $R_{W}$ and the right $Z$-module structure arising from $V_{Z}$, then $\mu$ is a left $W$ module map and right $Z$-module map.

Proof. We leave it to the reader to verify that $\eta$ and $\mu$ are well defined, and that (c) and (d) are true.

(a) Suppose $T$ is the direct sum of right $A$-modules $T_{1} \oplus T_{2}$. Then

$$
\mathrm{Hom}_{-X}(T, X)=\mathrm{Hom}_{-X}\left(T_{1}, X\right) \oplus \mathrm{Hom}_{-X}\left(T_{2}, X\right)
$$

and so

$$
\begin{aligned}
\operatorname{Hom}_{-Y}(S, U) \otimes_{X} \operatorname{Hom}_{-X}(T, X)= & {\left[\operatorname{Hom}_{-Y}(S, U) \otimes_{X} \operatorname{Hom}_{-X}\left(T_{1}, X\right)\right] } \\
& \oplus\left[\operatorname{Hom}_{-Y}(S, U) \otimes_{X} \operatorname{Hom}_{-X}\left(T_{2}, X\right)\right] .
\end{aligned}
$$


Similarly the range of $\eta$ decomposes

$$
\left[\mathrm{Hom}_{-Y}\left(T \otimes_{X} S, U\right)\right]=\left[\mathrm{Hom}_{-Y}\left(T_{1} \otimes_{X} S, U\right)\right] \oplus\left[\operatorname{Hom}_{-Y}\left(T_{2} \otimes_{X} S, U\right)\right]
$$

Letting $\eta$ denote the " $\eta$-map" for $T$ and letting $\eta_{i}$ denote the " $\eta$-map" for $T_{i}$, the following diagram commutes (verification left to the reader).

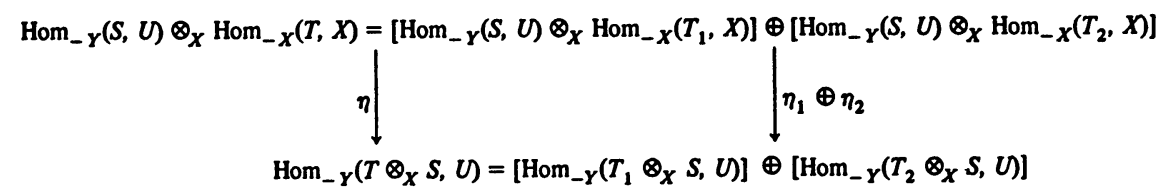

(*) Thus $\eta$ is bijective if and only if both $\eta_{1}$ and $\eta_{2}$ are bijective.

Now suppose $T=X$ as a right $X$-module. Then $\operatorname{Hom}_{-X}(T, X)=X$ as a left $X$-module, and the domain of $\eta$ is $\operatorname{Hom}_{-Y}(S, U) \otimes_{X} X=\operatorname{Hom}_{-Y}(S, U)$. The range of $\eta$ reduces to $\mathrm{Hom}_{-Y}\left(X \otimes_{X} S, U\right)=\mathrm{Hom}_{-Y}(S, U)$, and it is left to the reader to verify that $\eta$ reduces to the identity map. Hence $\eta$ is bijective when $T=X$.

When $T$ is a free right $X$-module with finite basis, it follows from (*) and the result for $T=X$ that $\eta$ is bijective. Again using (*), it follows that if $T$ is a direct summand of a free $X$-module with finite basis, then $\eta$ is bijective.

In other words, $\eta$ is bijective when $T$ is a finitely generated projective $X$ module. This concludes the proof of (a).

(b) is proved similarly. Q.E.D.

3.5. Duality Lemma. Suppose $X$ is a ring with modules ${ }_{X} R$ and $T_{X}$.

(a) $T^{*}=\operatorname{Hom}_{-X}(T, X)$ has the left $X$-module structure induced by ${ }_{X} X$ and ${ }^{*} R=\operatorname{Hom}_{X-}(R, X)$ has the right $X$-module structure induced by $X_{X}$. If $T$ is a finitely generated projective right $X$-module, then $T^{*}$ is a finitely generated projective left $X$-module. If $R$ is a finitely generated projective left $X$-module, then ${ }^{*} R$ is a finitely generated projective right $X$-module.

(b) $T^{*}$ has the left $X$-module structure of (a) which permits us to form ${ }^{*}\left(T^{*}\right)$. There is a natural map $\beta: T \rightarrow{ }^{*}\left(T^{*}\right)$ where for $t \in T, f \in T^{*}, \beta(t)(f)=$ $f(t)$. If ${ }^{*}\left(T^{*}\right)$ has the right $X$-module structure of $(\mathrm{a})$, then $\beta$ is a right $X$-module map.

(c) $\beta$ is bijective if $T$ is a finitely generated projective right $X$-module.

(d) If $T$ is a free right $X$-module with a basis of finite cardinality $n$, then $T^{*}$ is a free left $X$-module with a basis of cardinality $n$.

(e) ${ }^{*} R$ has the right $X$-module structure of (a) which permits us to form $(* R)^{*}$.

There is a natural map $\alpha: R \rightarrow\left({ }^{*} R\right)^{*}$ where, for $r \in R, g \in R^{*}, \alpha(r)(g)=$ 
$g(r)$. If $\left({ }^{*} R\right)^{*}$ has the left $X$-module structure of $(\mathrm{a})$, then $\alpha$ is a left $X$-module map.

(f) $\alpha$ is bijective if $R$ is a finitely generated projective left $X$-module.

(g) If $R$ is a free left $X$-module with a basis of finite cardinality $n$, then ${ }^{*} R$ is a free right $X$-module with a basis of cardinality $n$.

Proof. (b) and (d) are left to the reader. We sketch a proof of (c).

As in the proof of 3.4(a), if $T=T_{1} \oplus T_{2}$, a direct sum of right $X$-modules, then $\beta$ breaks up accordingly into $\beta_{1} \oplus \beta_{2}$, and $\beta$ is an isomorphism if and only if both $\beta_{1}$ and $\beta_{2}$ are isomorphisms.

In case $T=X_{X}$, then $T^{*}={ }_{X} X$, and ${ }^{*}\left(T^{*}\right)=X_{X}$, and $\beta$ corresponds to the identity map which is bijective. This, together with the behavior of $\beta$ for direct sums, shows that if $T$ is isomorphic to a finite direct sum of copies of $X_{X}$, then $\beta$ is an isomorphism. And so if $T$ is a direct summand of a finite direct sum of copies of $X_{X}$, then $\beta$ is an isomorphism. And all finitely generated projective right $X$-modules arise as direct summands of a finite direct sum of copies of $X_{X}$. This proves (c).

(e) is proved similarly.

(a), (d) and (g) have a similar direct sum argument type proof with no maps involved. Simply $T^{*} \cong T_{1}^{*} \oplus T_{2}^{*}$. Then if $T=B_{B}$, it follows $T^{*}={ }_{B} B$ etc. Q.E.D.

3.6. Example. Suppose $(C, \Delta, \epsilon)$ is a $B$-coring. Let the modules $R, S, T$ of 3.4 all be $C$. Let the modules $U, V$ of 3.4 both be $B$. Let the rings $W, X, Y, Z$ of 3.4 all be $B$. The diagonalization $\Delta: C \rightarrow C \otimes_{B} C$ is then a map $\delta: C \rightarrow$ $T \otimes_{X} S$ and $\Delta$ is a left $Z$ and right $Y$-module map if ${ }_{Z} C_{Y}={ }_{B} C_{B}$. This $\delta$ induces $\operatorname{Hom}(\delta, U): \operatorname{Hom}_{-Y}\left(T \otimes_{X} S, U\right) \rightarrow \operatorname{Hom}_{-Y}(C, U)$ which is a left $W$ and right $Z$-module map. Forming the composite $\operatorname{Hom}(\delta, U) \eta$ gives a map $\operatorname{Hom}_{-Y}(S, U)$ $\otimes_{X} \mathrm{Hom}_{-X}(T, X) \rightarrow \mathrm{Hom}_{-Y}(C, U)$, which is a map $C^{*} \otimes_{B} C^{*} \rightarrow C^{*}$. The reader can check that this map is precisely the opposite multiplication map of 3.2(b). That is $f \otimes g \rightarrow$ product $g f$ as described in 3.2(b). Thus the dual algebra structure on $C^{*}$ given in 3.2(b) is opposite to the structure induced by 3.4(a). Similarly the dual algebra structure on ${ }^{*} C$ given in $3.2(a)$ is opposite to the structure induced by $3.4(\mathrm{~b})$.

With suitable "finitely-generated-projective-module" type assumptions, the situation $\varphi: B \rightarrow E$, where $B$ and $E$ are rings and $\varphi$ a ring antimap, can be dualized to obtain a $B$-coring. Of course there is left duality and right duality.

Suppose $B$ and $E$ are rings and $\varphi: B \rightarrow E$ a ring antimap. We wish to apply 3.3 and 3.4 with

all the rings $W, X, Y, Z$ equal to $B$,

the modules $R, S, T$ equal to $E$,

the modules $U, V$ equal to $B$.

$B$ has the usual $B$-bimodule structure. 
The right and left $B$-module structures on $E$ are induced by $\varphi$ as follows: For $b \in B, e \in E$,

$$
b \cdot e \equiv e \varphi(b), \quad e \cdot b \equiv \varphi(b) e, \quad \text { product in } E .
$$

This gives $E$ left and right $B$-module structures since $\varphi$ is a ring antihomomorphism. And $E$ is a $B$-bimodule.

Note that if $C$ is a $B$-coring and $E={ }^{*} C$ (respectively $C^{*}$ ), and $\varphi=\lambda$ (respectively $\rho$ ) as in 3.2, then the $B$-bimodule structure on $E$ induced by $\varphi$ agrees with the $B$-bimodule structure where ${ }_{B}^{*} C$ is induced by $C_{B}$ and ${ }^{*} C_{B}$ is induced by $B_{B}$ (respectively, ${ }_{B} C^{*}$ is induced by ${ }_{B} B$ and $C_{B}^{*}$ is induced by ${ }_{B} C$.) Hence $E$ has the appropriate $B$-module structure to apply 3.5 .

3.7. Dual Coring Theorem. (a) In the above setting, if $E^{*}=$ $\operatorname{Hom}_{-B}(E, B)$ has the left $B$-module structure as in 3.5(a), and the right B-module structure induced by ${ }_{B} E$, and $E$ is finitely generated and projective as a right $B$ module, then there is a unique $B$-coring structure on $E^{*}$ whereby the bijection $\beta: E \rightarrow{ }^{*}\left(E^{*}\right) 3.5(\mathrm{~b})$ is a ring isomorphism. Here ${ }^{*}\left(E^{*}\right)$ has the ring structure of 3.2(a).

Moreover the diagram

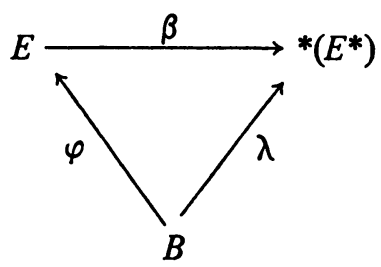

commutes, where $\lambda$ is defined in $3.2(\mathrm{c})$.

(b) In the above setting, if ${ }^{*} E=\operatorname{Hom}_{B-}(E, B)$ has the right $B$-module structure as in 3.5(a), and the left $B$-module structure induced by $E_{B}$, and $E$ is finitely generated and projective as a left $B$-module, then there is a unique $B$ coring structure on ${ }^{*} E$ whereby the bijection $\alpha: E \rightarrow\left({ }^{*} E\right)^{*} 3.5(\mathrm{~b})$ is a ring isomorphism. Here $\left({ }^{*} E\right)^{*}$ has the ring structure of $3.2(\mathrm{~b})$.

Moreover the diagram

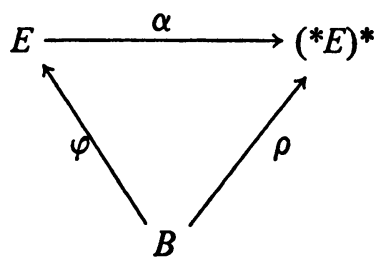

commutes, where $\rho$ is defined in $3.2(\mathrm{~d})$.

Proof. (a) Define $\epsilon: E^{*} \rightarrow B$ by $\epsilon(f)=f(1), f \in E^{*}, 1 \in E$. Then it is easily checked that $\epsilon$ is a $B$-bimodule map. 
With the choices of $W, X, Y, Z, S, T, U$ indicated just before this theorem, the map $\eta$ of 3.4(a) is an isomorphism. Thus $\eta^{-1}$ is an isomorphism

$$
\eta^{-1}: \operatorname{Hom}_{-Y}\left(T \otimes_{X} S, U\right) \rightarrow \operatorname{Hom}_{-Y}(S, U) \otimes_{X} \operatorname{Hom}_{-X}(T, X),
$$

and by $3.4(\mathrm{c}), \eta^{-1}$ is a left $W$ and right $Z$-module map. Since $T=E=S$, the map tlum: $E \otimes_{B} E \rightarrow E, e \otimes f \rightarrow f e$, induces

$$
T \otimes_{X} S \rightarrow E
$$

which is a left $Z$-module map and right $Y$-module map. Hence ${ }_{B} E_{B}$ becomes ${ }_{Z} E_{Y}$. Thus (**) induces

$$
\mathrm{Hom}_{-Y}(E, U) \rightarrow \mathrm{Hom}_{-Y}\left(T \otimes_{X} S, U\right)
$$

which is a left $W$-module map and right $Z$-module map.

Composing the maps at (*) and (***) gives the map

$$
\mathrm{Hom}_{-Y}(E, U) \rightarrow \mathrm{Hom}_{-Y}(S, U) \otimes_{X} \mathrm{Hom}_{-X}(T, X) .
$$

With the choices of $W, X, Y, Z, S, T, U,\left(\begin{array}{c}* * \\ * *\end{array}\right)$ is a bimodule map, $E^{*} \rightarrow E^{*} \otimes_{B}$ $E^{*}$, denoted $\Delta$.

That $\left(E^{*}, \Delta, \epsilon\right)$ satisfies the necessary commutative diagrams to be a $B$-coring follows from commutative diagrams that $E$ satisfies and the duality established.

That $\beta: E \rightarrow^{*}\left(E^{*}\right) 3.5(b)$ is a ring isomorphism, and $\beta \varphi=X$ is left to the reader to verify. The uniqueness of the coring structure is also left to the reader to verify.

(b) goes similarly. Q.E.D.

4. Jacobson-Bourbaki-Hochschild. Throughout this section $B$ is a division ring and End $B$ denotes $\operatorname{End}_{z} B$, the ring of additive group endomorphisms of $B$. $l$ and $r$ denote the maps

$$
B \rightarrow \text { End } B, \quad b \rightarrow b^{l}, \quad \beta \rightarrow \beta^{r}
$$

where $b, \beta \in B, b^{l}(\beta)=b \beta=\beta^{r}(b)$.

Since $B$ has a unit, $l$ and $r$ are injective. $\operatorname{Im} l$ is denoted $B^{l}$, and $\operatorname{Im} r$ is denoted $B^{r}$. Since $l$ is a ring map, $B \cong B^{l}$ as rings. Since $r$ is a ring antihomomorphism, $B^{r}$ is isomorphic to the opposite ring of $B$.

It is amusing to verify that $B^{r}$ is the centralizer of $B^{l}$ in End $B$ and $B^{l}$ the centralizer of $B^{r}$. This is left to the reader.

Let $F$ be a subring of End $B$ with End $B \supset F \supset B^{r}$. Since $F \supset B^{r}$, the centralizer of $F$ lies in $B^{l}$. Let $A$ denote $\{a \in B \mid f(a b)=a f(b), b \in B, f \in F\}$.

It is easily verified (and left to the reader) that

(i) $A$ is a subring of $B$ and $A$ is a division ring,

(ii) $A^{l}=\left\{a^{l}\right\}_{a \in A}$ is the centralizer of $F$ in End $B$ 
(iii) $B^{r} \subset F \subset$ End $_{A-} B \subset$ End $B$,

(iv) $A=\{b \in B \mid f(b)=b f(1), f \in F\}$.

4.1. THEOREM. If $F$ has finite dimension as a right $B$-module or $B$ has finite dimension as a left $A$-module, i.e. either $\operatorname{dim}_{B} F_{B}<\infty$ or $\infty>\operatorname{dim}_{A} A$, then both are finite and equal and $F=$ End $_{A-} B$.

Proof. The idea behind the proof is to show that $F^{*}$ equals or is naturally isomorphic to the $B$-coring $B \otimes_{A} B$ of 1.2. Then it will follow from 3.3 and 3.7 that $F={ }^{*}\left(F^{*}\right)=\operatorname{End}_{A-B} B$.

Now the proof actually begins.

Let $X$ be an $A$-basis for ${ }_{A} B$ and let $\left\{\delta_{x}\right\}_{x \in X} \subset$ End $_{A-} B$ be determined by

$$
\delta_{x}(y)=\left\{\begin{array}{ll}
0, & x \neq y, \\
1, & x=y,
\end{array} \quad x, y \in X .\right.
$$

Then $\left\{\delta_{x}\right\}$ is a $B$-linearly independent set for $\left(\operatorname{End}_{A-} B\right)_{\dot{B}}$ and $\left\{\delta_{x}\right\}$ is a $B$-basis for $\left(\operatorname{End}_{A-} B\right)_{B}$ if $\operatorname{dim}_{A}{ }_{A} B<\infty$. Thus

$$
\operatorname{dim}_{B}\left(\operatorname{End}_{A-} B\right)_{B}= \begin{cases}\operatorname{dim}_{A} A^{B} & \text { if } \operatorname{dim}_{A} B<\infty, \\ \infty & \text { if } \operatorname{dim}_{A} B=\infty .\end{cases}
$$

Since $F \subset$ End $_{A-} B$, it follows that $\operatorname{dim}_{B} F_{B}<\infty$ if $\operatorname{dim}_{A}{ }_{A} B<\infty$.

Thus assume for the rest of the proof that $\operatorname{dim}_{B} F_{B}<\infty$.

For $f \in F, \Sigma b_{i} \otimes \beta_{i} \in B \otimes_{A} B$, define

$$
\left\langle f, \sum b_{i} \otimes \beta_{i}\right\rangle \equiv \sum b_{i} f\left(\beta_{i}\right) \in B .
$$

For fixed $\Sigma b_{i} \otimes \beta_{i} \in B \otimes_{A} B$, the function $\left\langle, \Sigma b_{i} \otimes \beta_{i}\right\rangle$ lies in $F^{*}$, and for fixed $f \in F$, the function $\langle f$,$\rangle lies in *\left(B \otimes_{A} B\right)$.

For $0 \neq f \in F$ let $b \in B$ with $f(b) \neq 0$. Then

$$
K: B \otimes_{A} B \rightarrow F^{*}, \quad \sum b_{i} \otimes \beta_{i} \rightarrow\left\langle, \sum b_{i} \otimes \beta_{i}\right\rangle
$$

has dense image in $F^{*}$ since $\langle f, 1 \otimes b\rangle=f(b) \neq 0$.

Using 3.5 and $\operatorname{dim}_{B} F_{B}<\infty$, it follows, just like in commutative linear algebra, that $K$ is surjective.

Dualizing once again yields $*\left(B \otimes_{A} B\right) \stackrel{{ }^{*} K}{\longleftarrow} *\left(F^{*}\right)$.

Identifying ${ }^{*}\left(F^{*}\right)$ with $F$ as $a$ ring by $3.7(\mathrm{a})$ and identifying $*\left(B \otimes_{A} B\right)$ with $\operatorname{End}_{A-} B$ as a ring by 3.3, then ${ }^{*} K$ reduces to the inclusion.

Thus * $K$ is a ring map which implies that $K$ is a $B$-coring map. (This type of duality result we are using without including a proof.) 
Let $g=1 \otimes 1 \in B \otimes_{A} B$, the distinguished grouplike element of 1.9(a).

Let $c=K(g)$, a grouplike in $F^{*}$, since $K$ is a coring map. Since $B \otimes_{A} B=$ $B g B$, it follows that $F^{*}=B c B$ since $K$ is a surjective $B$-bimodule map.

If $A=\{a \in B \mid a c=c a\}$, then, by $2.2, K$ is injective and, hence, an isomorphism. This implies that ${ }^{*} K$ is an isomorphism. By (4.3) it follows that $F=\operatorname{End}_{A}-B$. Then by (4.2) and the assumption $\operatorname{dim}_{B} F_{B}<\infty$, it follows that

$$
\infty>\operatorname{dim}_{A} B=\operatorname{dim}_{B}\left(\text { End }_{A-} B\right)_{B}=\operatorname{dim}_{B} F_{B},
$$

which would conclude the proof.

$$
c=K(g)=\langle, 1 \otimes 1\rangle \text {. For } b \in B, c b=\langle, 1 \otimes b\rangle \text { and } b c=\langle, b \otimes 1\rangle \text {. }
$$

Thus for $f \in F,(b c)(f)=b f(1),(c b)(f)=f(b)$.

Hence by (iv)-immediately above $4.1-A=\{b \in B \mid b c=c b\}$. Q.E.D.

In [3] Hochschild studies an object other than $\operatorname{End}_{A-B}$. Suppose $Z$ is a division ring intermediate between $A$ and $B, A \subset Z \subset B$. Then one can consider $\operatorname{Hom}_{A-}(B, Z)$, which of course is a right ideal in $\operatorname{End}_{A-}(B)$ if we take

$$
\operatorname{Hom}\left(B \text {, inclusion): } \operatorname{Hom}_{A-}(B, Z) \rightarrow \operatorname{Hom}_{A-}(B, B)\right.
$$

as an inclusion. As a right ideal, $\operatorname{Hom}_{A-}(B, Z)$ has a product structure but not a multiplicative identity (in general). Although $\operatorname{Hom}_{A-}(B, Z)$ is a right ideal, it is a left $Z$-submodule via $r$ of $\left(\operatorname{End}_{A-} B\right)_{B} r \supset Z^{r} \cdot \operatorname{Hom}_{A-}(B, Z)$ is the $Z$-dual, Hom $_{Z-}(, Z)$, of $Z \otimes_{A} B$. Such a $Z \otimes_{A} B$ is a left $Z$-module and right $B$-module. There is a natural diagonal map

$Z \otimes_{A} B \rightarrow Z \otimes_{A} B \otimes_{A} B=\left(Z \otimes_{A} B\right) \otimes_{Z}\left(Z \otimes_{A} B\right), \quad z \otimes b \rightarrow z \otimes 1 \otimes b$,

but there is no natural counit making $Z \otimes_{A} B$ into a $Z$-coring. The theory of such coassociative corings without counit could be developed and would yield the Galois theorem to which [3, Theorem 2.1, p. 447] is dual in the finite case.

\section{BIBLIOGRAPHY}

1. H. Cartan, Théorie de Galois pour les corps non commutatifs, Ann. Sci. École Norm. Sup. (3) 64 (1947), 59-77. MR 9, 325.

2. J. Dieudonné, Linearly compact spaces and double vector spaces over $s$ fields, Amer. J. Math. 73 (1951), 13-19. MR 12, 476.

3. G. Hochschild, Double vector spaces over division rings, Amer. J. Math. 71 (1949), 443-460. MR 10, 676.

4. N. Jacobson, An extension of Galois theory to non-normal and non-separable fields, Amer. J. Math. 66 (1944), 1-29. MR 6, 35.

5. - A note on division rings, Amer. J. Math. 69 (1947), 27-36. MR 9, 4.

6. - Lectures in abstract algebra. Vol. 3: Theory of fields and Galois theory, Van Nostrand, Princeton, N. J., 1964. MR 30 \#3087.

7. M. E. Sweedler, Hopf algebras, Math. Lecture Note Series, Benjamin, New York, 1969. MR 40 \#5705.

DEPARTMENT OF MATHEMATICS, CORNELL UNIVERSITY, ITHACA, NEW YORK 